\title{
二氧化碳参与的自由基型烯烃双官能团化反应
}

\author{
张振 $a, b$ 龚莉 ${ }^{b}$ 周晓渝 ${ }^{b}$ 颜思顺 ${ }^{b}$ 李静*,b 余达刚 $*, b$ \\ ( ${ }^{a}$ 成都大学药学与生物工程学院 农业农村部杂粮加工重点实验室 成都 610106) \\ $\left({ }^{b}\right.$ 四川大学化学学院 绿色化学与技术教育部重点实验室 成都 610064)
}

\begin{abstract}
摘要 二氧化碳 $\left(\mathrm{CO}_{2}\right)$ 是一种理想的 $\mathrm{C} 1$ 合成子. 利用其参与化学转化合成羧酸和含羰基杂环等具有高附加值的产品, 具有重要意义. 另一方面, 烯烃的双官能团化反应是有机合成化学中的一类重要反应，可以将简单易得的烯烃快速高 效地转化为结构多样性的重要化合物. 然而, 由于 $\mathrm{CO}_{2}$ 反应活性较低, 而且烯烃官能团化反应的选择性难以控制, $\mathrm{CO}_{2}$ 参与的烯烃双官能团化反应具有较高的挑战性. 近年来, 自由基化学的蓬勃发展为该类反应的开发提供了新的策略, 实现了一些重要转化反应. 基于此, 从 $\mathrm{CO}_{2}$ 参与烯烃的氧-烷基化反应、碳羧基化反应、硅㜋基化反应、硫羧基化反应 以及双羧基化反应等反应入手, 全面总结和深入分析了最近几年 $\mathrm{CO}_{2}$ 参与的自由基型烯烃双官能团化反应进展; 在介 绍上述进展的同时，重点阐述了其可能经历的四类自由基化学历程. 最后对该领域的未来发展方向进行了展望，希望 为该领域的进一步发展提供一些思路.
\end{abstract}

关键词 二氧化碳; 烯烃; 双官能团化; 自由基; 可见光催化

\section{Radical-Type Difunctionalization of Alkenes with $\mathrm{CO}_{2}$

\begin{tabular}{|c|c|}
\hline ng, Zhen ${ }^{a, b}$ & $\begin{array}{l}\text { Gong, } \text { Li }^{b} \\
\text { Li, Jing*, }\end{array}$ \\
\hline
\end{tabular}

( ${ }^{a}$ Key Laboratory of Coarse Cereal Processing (Ministry of Agriculture and Rural Affairs), College of Pharmacy and Biological Engineering, Chengdu University, Chengdu 610106)

$\left({ }^{b}\right.$ Key Laboratory of Green Chemistry \& Technology of Ministry of Education, College of Chemistry, Sichuan University, Chengdu 610064)

\begin{abstract}
CO}_{2}$ is an ideal $\mathrm{C} 1$ source in chemical transformations. It is of great significance to utilize $\mathrm{CO}_{2}$ in chemical conversion to synthesize high value-added compounds, including carboxylic acids and carbonyl-containing heterocycles. On the other hand, the difunctionalization of olefins is an important organic reaction, which can efficiently convert easily available olefins into important compounds with structural diversity. However, due to the low reactivity of $\mathrm{CO}_{2}$ and the difficulty in controlling the selectivity, the difunctionalization of olefins with $\mathrm{CO}_{2}$ is highly challenging. Recently, the significant progress of radical chemistry has provided new strategies and promoted the development of novel transformations in this field. This Perspective summarizes the recent progress of the radical-type difunctionalization of olefins with $\mathrm{CO}_{2}$, including the oxy-alkylation, carbocarboxylation, silacarboxylation, thiocarboxylation, and dicarboxylation of alkenes with $\mathrm{CO}_{2}$. At the same time, we also highlight the mechanism with radicals and four kinds of pathways are proposed: (1) Free radicals attack olefins to form new carbon radical intermediates. The radicals are then oxidized to form carbocations, which are further captured by carbonates or carbamates. It is also possible for direct $\mathrm{C}-\mathrm{O}$ bonding reaction or sequent $\mathrm{C}-\mathrm{I}$ and $\mathrm{C}-\mathrm{O}$ bonds formation. (2) The new carbon radical intermediates, in-situ generated through attack of alkenes with radicals, are reduced via single electron transfer into carbanions, which could attack $\mathrm{CO}_{2}$ to form $\mathrm{C}-\mathrm{C}$ bonds. (3) $\mathrm{CO}_{2}$ is reduced into $\mathrm{CO}_{2}$ radical anions in the highly reductive reaction conditions. Once generated, the $\mathrm{CO}_{2}$ radical anions might attack olefins to form carboxylate bearing more stable carbon radical intermediates (such as benzylic ones) and further form $\mathrm{C}-\mathrm{C}$ bonds or carbon-heteroatom bonds. (4) Olefins are reduced via single electron transfer into alkenyl free radical anions in the highly reductive reaction conditions. These anions may attack $\mathrm{CO}_{2}$ to form carboxylate bearing carbon radical intermediates and are further reduced to generate carbanions. Finally they may attack another $\mathrm{CO}_{2}$ to form succinic acid derivatives. We point out the challenges and predict the future development in the field, including the more challenging substrates, more reaction types, better selectivities, and deeper mechanistic understanding.

Keywords carbon dioxide; olefin; difunctionalization; radical; visible-light photoredox catalysis
\end{abstract}

* E-mail: jingli@scu.edu.cn; dgyu@scu.edu.cn; Tel.: 028-85414510

Received June 12, 2019; published July 12, 2019.

Project supported by the "973" Project from the Ministry of Science and Technology of China (No. 2015CB856600), the National Natural Science Foundation of China (Nos. 21822108, 21801025), the Fok Ying Tung Education Foundation (No. 161013) and the Fundamental Research Funds for the Central Universities.

项目受科技部 “973” (No. 2015CB856600)、国家自然科学基金(Nos. 21822108, 21801025)、教育部霍英东基金(No. 161013)和中央高校基本业务费资 助. 


\section{1 引言}

二氧化碳 $\left(\mathrm{CO}_{2}\right)$ 是空气的重要组分, 也是重要的 $\mathrm{C} 1$ 资源. 由于其无毒、廉价易得、储量丰富且可循环再生 等特点, $\mathrm{CO}_{2}$ 是合成化学中的理想 $\mathrm{C} 1$ 合成子 ${ }^{[1]}$. 利用 $\mathrm{CO}_{2}$ 参与化学转化, 通过形成 $\mathrm{C}-\mathrm{C}$ 和 $\mathrm{C}-\mathrm{O}$ 等化学键, 可以高效构筑一系列具有良好生物活性的分子骨架和 功能材料等具有高附加值的产品, 具有潜在的工业应用 前景. 因此, 基于 $\mathrm{CO}_{2}$ 这一 $\mathrm{C} 1$ 合成子的化学转化一直 备受关注 ${ }^{[2]}$ 并取得了一系列的成果: 例如, 通过 $\mathrm{CO}_{2}$ 与 过渡金属催化 ${ }^{[3]}$ 、光催化 ${ }^{[4]}$ 、电催化 ${ }^{[5]}$ 、碱促进 ${ }^{[6]}$ 、离子 液体等的结合 ${ }^{[7]}$, 实现多种羧基化、羰基化等产物的合 成; 通过对二氧化碳的还原, 实现化合物甲基化、甲酰 化 ${ }^{[8]}$ 的转化或直接将二氧化碳转化为甲酸 ${ }^{[9]}$ 等能源性物 质; 更奇特的是, 利用二氧化碳的特殊性质, 将二氧化 碳作为化学辅助试剂, 实现化合物的氧化、官能团 化 ${ }^{[10]}$.

另一方面, 烯烃是有机合成中最重要的合成前体之 一, 取代类型多样, 且来源广泛, 易于制备. 此外, 烯烃 参与的官能团化反应种类繁多, 且选择性可以调控, 易 于快速合成具有重要官能团的分子, 是有机合成化学的 重要工具 ${ }^{[11]}$. 其中, $\mathrm{CO}_{2}$ 作为 $\mathrm{C} 1$ 合成子参与的烯烃官能 团化反应, 可以直接合成羧酸、噁唑啉酮和醇类等重要 有机化合物. 近年来人们在该领域进行了大量研究, 开 发了多样的催化体系, 如过渡金属催化、光催化、电催 化和有机小分子催化等 ${ }^{[12]}$.

$\mathrm{CO}_{2}$ 参与的烯烃官能团化反应主要分为烯烃单官能 团化反应和双官能团化反应. 其中, $\mathrm{CO}_{2}$ 参与的烯烃单 官能团化反应最为常见, 主要包括发展较为成熟的氢羧 基化反应 ${ }^{[13]}$ 和还原羟甲基化 ${ }^{[14]}$ 等反应. 与之相比, $\mathrm{CO}_{2}$ 参与的烯烃双官能团化反应, 例如碳羧基化反应、硫羧 基化反应和硅羧基化反应等, 可以同时构建多个化学 键, 形成结构多样、高度官能团化的有机化合物, 具有 更高的价值.

然而, 相比于烯烃单官能团化反应而言, $\mathrm{CO}_{2}$ 参与 的烯烃双官能团化反应报道相对较少, 具有较大的挑战 性. 首先, 由于其热力学稳定性和动力学惰性, $\mathrm{CO}_{2}$ 是一 种反应活性较低的亲电试剂, 不易直接与烯烃反应, 甚 至和一些有机金属试剂都不容易反应(如: 需要高温高 压促进芳基钯物种和 $\mathrm{CO}_{2}$ 反应), 因此需要开发高活性 体系活化烯烃(如: 将烯烃转化为高活性的烷基金属物 种或者碳负离子 ${ }^{[12 \mathrm{~d}, 12 \mathrm{~N}, 13]}$, 进而进攻 $\mathrm{CO}_{2}$ ) 或者 $\mathrm{CO}_{2}$ (如: 通过单电子还原 $\mathrm{CO}_{2}$ 形成自由基负离子, 然后直接进攻 烯烃 $\left.{ }^{[5]}\right)$; 其次, $\mathrm{CO}_{2}$ 参与的烯烃双官能团化反应属于多 组分反应, 还存在着诸多选择性问题: (1)化学选择性. 如前所述, $\mathrm{CO}_{2}$ 参与的烯烃氢羧基化反应是 $\mathrm{CO}_{2}$ 参与的 烯烃官能团化反应中研究最多的形式. 该反应主要经由 烯烃对金属氢物种的插入形成亲核性的有机金属物种, 进而对 $\mathrm{CO}_{2}$ 进攻得到羧酸衍生物. 相比而言, 烯烃对其
他金属物种中的碳金属键或者杂原子金属键进行插入， 进而与 $\mathrm{CO}_{2}$ 反应的研究较少, 其原因可能在于: 类似于 烯烃的碳碳双键, $\mathrm{CO}_{2}$ 中的碳氧双键也可以与反应体系 中的金属物种直接发生背景反应(如迁移插入反应、氧 化环金属化反应等); (2) 区域选择性. 在 $\mathrm{CO}_{2}$ 参与的烯烃 双官能团化反应中，除了使用结构对称的烯烃或者发生 双羧基化反应外, 其他反应类型均存在成键的区域选择 性问题; (3)对映选择性. 相比于化学选择性和区域选择 性, $\mathrm{CO}_{2}$ 参与的烯烃双官能团化反应中的对映选择性控 制则更加困难. 尽管有很多尝试, $\mathrm{CO}_{2}$ 参与的不对称碳 碳键形成反应还存在巨大挑战, 目前能够实现高对映选 择性的例子还很少.

综上所述, $\mathrm{CO}_{2}$ 参与的烯烃双官能团化反应存在诸 多挑战. 尽管如此, 人们通过大量尝试, 开发了一些新 型策略, 尤其是通过近年来蓬勃发展的自由基化学, 利 用高活性的自由基参与反应，在相对温和的条件下实现 了多种 $\mathrm{CO}_{2}$ 参与的烯烃双官能团化反应. 根据具体历 程, 我们将 $\mathrm{CO}_{2}$ 参与的自由基型烯烃双官能团化反应分 为四类: (1)自由基进攻烯烃形成新的碳自由基中间体, 随后被单电子氧化形成碳正离子, 再进一步被碳酸盐或 者氨基甲酸盐捕获, 或者直接发生氧化型的碳氧成键反 应，又或者先发生自由基擭碘形成烷基碘，进而被氨基 甲酸盐捕获形成碳氧键(图 1, path A); (2)自由基进攻烯 烃形成新的碳自由基中间体后，被单电子还原为碳负离 子, 进而进攻 $\mathrm{CO}_{2}$ 形成碳碳键(图 1, path B); (3) $\mathrm{CO}_{2}$ 在 强还原体系中被还原为二氧化碳自由基负离子, 进攻烯 烃形成较为稳定的碳自由基中间体，进一步形成碳碳键 或者碳杂原子键(图 1, path C); (4)烯烃在强还原体系中 被单电子还原为烯基自由基负离子, 随后进攻 $\mathrm{CO}_{2}$ 形成 较为稳定的碳自由基中间体, 进一步被单电子还原为碳 负离子, 最后进攻第二分子的 $\mathrm{CO}_{2}$ 形成丁二酸类衍生物 (图 1, path D). 本文将重点围绕最近几年 $\mathrm{CO}_{2}$ 参与的这 几类自由基化学历程的烯烃双官能团化反应进行总结, 并对该领域的进一步发展进行展望.

\section{$2 \mathrm{CO}_{2}$ 参与的自由基型烯烃双官能团化反应}

\section{$2.1 \mathrm{CO}_{2}$ 参与的噁唑啉酮类化合物的合成}

噁唑啉酮广泛存在于药物分子当中. 例如, 利奈唑 酮(Linezolid)、美他沙酮(Skelaxin)和佐米曲普坦(Zolmitriptan)等都含有噁唑啉酮结构. 另外, 噁唑啉酮也是有 机合成化学中广泛使用的反应中间体和手性辅基 ${ }^{[16]}$. 对于此类化合物的制备传统上多采用光气、氨基甲酸酯 等与 $\beta$-氨基乙醇、 $\beta$-卤代乙醇、1,2-乙二醇等反应. 而鉴 于该类化合物的重要性, 有必要开拓更为多样有效的制 备方式, 其中, 通过烯丙胺与 $\mathrm{CO}_{2}$ 实现烯烃双键的双官 能团化反应制备噁坐啉酮不仅能够有效地利用 $\mathrm{CO}_{2}$ 这 一优良的 $\mathrm{C} 1$ 合成子，同时烯丙胺合成方法众多，可以 更好的实现噁唑啉酮的多样化制备. 但是通过烯丙胺 


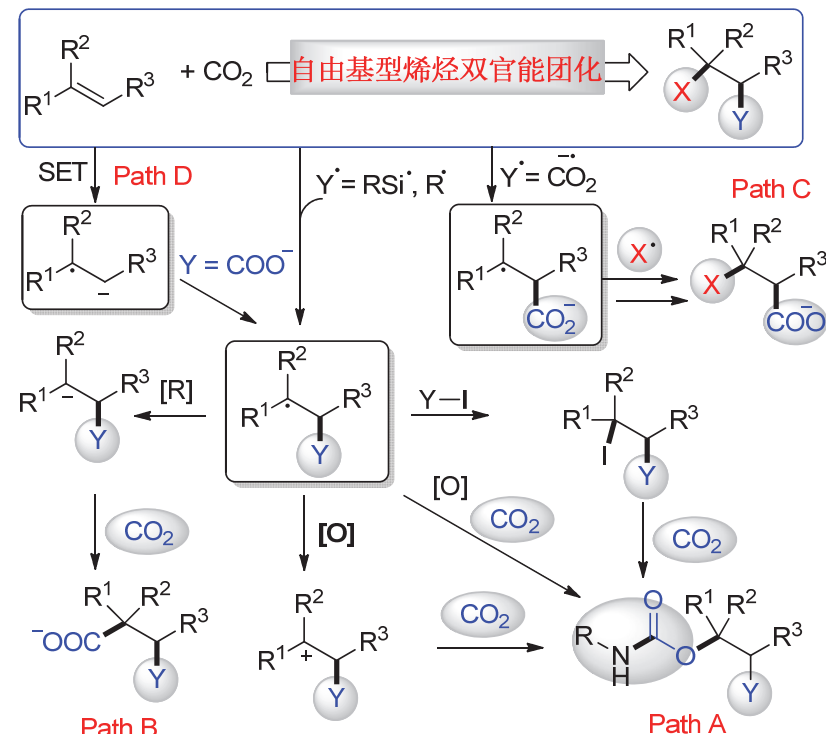

Path B

与 $\mathrm{CO}_{2}$ 实现烯烃双键的双官能团化反应制备噁唑啉酮 的方法目前还很有限, 取代类型单一, 不能满足药物开 发对多样性的需求. 针对该问题, 近年来人们通过自由 基化学, 利用自由基前体、烯丙胺与 $\mathrm{CO}_{2}$ 参与的三组分 反应成功实现噁唑啉酮合成的同时，可以引入多种类型 的取代基团, 具有重要的合成价值.

\subsection{1 烯丙胺的氧一三氟甲基化}

2016年, 我们课题组 ${ }^{[17]}$ 以 Togni 试剂为自由基前体, 首次报道了铜催化的 $\mathrm{CO}_{2}$ 参与的烯丙胺的氧一三氟甲基 化反应, 得到了一系列含 $\mathrm{CF}_{3}$ 基团的重要噁唑啉酮类化 合物(图 2). 在这类多组分反应中, 主要存在着以下挑 战: (1)氮一三氟甲基化与氧一三氟甲基化之间的化学选择 性. Sodeoka 和 Cho 分别报道了在温和条件下三氟甲基 化试剂参与的烯丙胺中碳碳双键的氮三氟甲基化反应. 反应中如何有效地生成氨基甲酸中间体并优先于 $\mathrm{C}-\mathrm{N}$ 键形成 $\mathrm{C}-\mathrm{O}$ 键, 对该反应至关重要; (2) endo 和 exo 环 化之间的区域选择性。除化学选择性外，环化步骤

图 1 二氧化碳参与的自由基型烯烃双官能团化反应

Figure 1 Radical-type difunctionalization of alkenes with $\mathrm{CO}_{2}$<smiles>[R]NC([R])C([R])=C([R])[R]</smiles><smiles>[R]C1N([R])C(=O)OC1([R])C([R])([R])F</smiles>

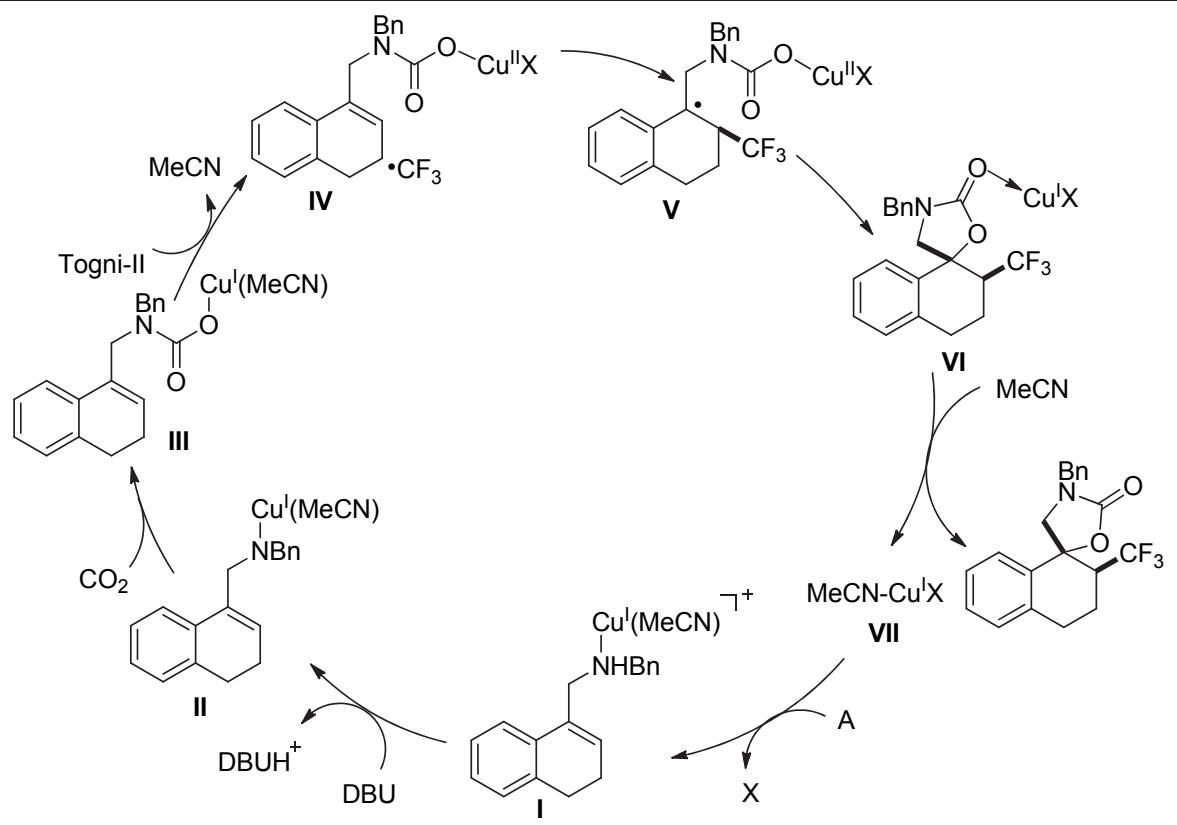

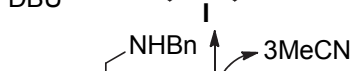

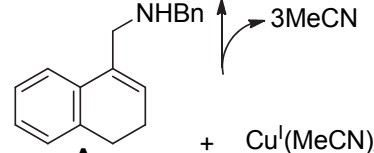

A $+\mathrm{Cu}^{\prime}(\mathrm{MeCN})_{4}$<smiles>Cc1cccc(C2(CC(F)(F)F)CN(Cc3ccccc3)C(=O)O2)c1</smiles>

$1,90 \%$

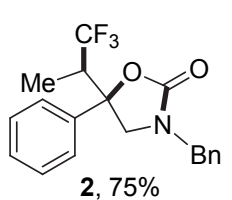

2, $75 \%$<smiles>O=C(NCC1(c2ccccc2)CCCc2ccccc21)C(F)(F)F</smiles>

(士) $3,78 \%^{\mathrm{Bn}}$

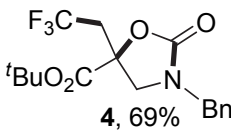

图 $2 \mathrm{CO}_{2}$ 参与的烯丙胺的氧一三氟甲基化

Figure 2 Selective oxytrifluoromethylation of allylamines with $\mathrm{CO}_{2}$ 
必须具有区域选择性才能以良好的收率得到单一杂环;

(3) 产物的稳定性问题. $\mathrm{CF}_{3}$ 的 $\alpha$ 位上的 $\mathrm{H}$ 具有较强的酸 性, 碱性条件下可能会导致 2-噁唑啉酮通过脱除一分子 $\mathrm{CO}_{2}$ 而发生分解反应. 为克服以上挑战, 我们组经过大 量条件篮选后, 成功的在 $101 \mathrm{kPa}$ 的 $\mathrm{CO}_{2}$ 氛围中和室温 下高选择性地实现了 $\mathrm{CO}_{2}$ 参与的烯丙胺的氧一三氟甲基 化反应. 该反应底物范围广、官能团兼容性好、条件温 和、整体反应收率高, 且能够实现克级规模反应, 产品 易于衍生.

随后, 为了对该反应有更为深入的理解, 我们课题 组与蓝宇课题组 ${ }^{[18]}$ 合作, 通过密度泛函理论(DFT)计算 详细地研究了该反应的机理. 研究结果表明, 烯丙胺 $\mathrm{A}$ 中的 $\mathrm{N}$ 原子与 $\mathrm{Cu}(\mathrm{I})$ 催化剂前体配位形成络合物 $\mathbf{I}$, 该络 合物在碱的作用下失去质子形成络合物 II. 计算证明去 质子这一步为整个催化循环的决速步. 随后, $\mathrm{CO}_{2}$ 插入 到 $\mathrm{N}-\mathrm{Cu}$ 键中形成中间体 III, 该中间体中的亚铜离子 进一步与亲电性的 Togni 试剂发生单电子转移并形成氨 基甲酸铜(II)中间体 IV 和游离的三氟甲基自由基. 该三 氟甲基自由基可进一步进攻烯烃并形成稳定的茮基自 由基物种 $\mathbf{V}$, 经过分子内的自由基-自由基偶联得到螺 环铜物种 VI, VI 经过配体交换最终得到氧一三氟甲基化 产物并释放出活性 $\mathrm{Cu}(\mathrm{I})$ 中间体 I. 另外, 计算表明, 缺 电子 $\mathrm{Cu}(\mathrm{II})$ 中心和亲电的三氟甲基自由基不能反应产生 铜(III)中间体; 前线分子轨道理论分析表明, 与 Togni 试 剂 II 发生单电子转移的是中性的 $\mathrm{Cu}(\mathrm{I})$ 物种, 而不是阳 离子 $\mathrm{Cu}(\mathrm{I})$ 物种; 此外, 非对映选择性可能源于大位阻 的三氟甲基和羰基部分之间的空间位阻排斥.

在此基础上, 我们课题组和蓝宇课题组 ${ }^{[19]}$ 又进一 步合作实现了铜催化的 $\mathrm{CO}_{2}$ 参与的自由基型去芳构化 反应(图 3). 多种吲哚和呋喃可以发生氧一三氟甲基化的

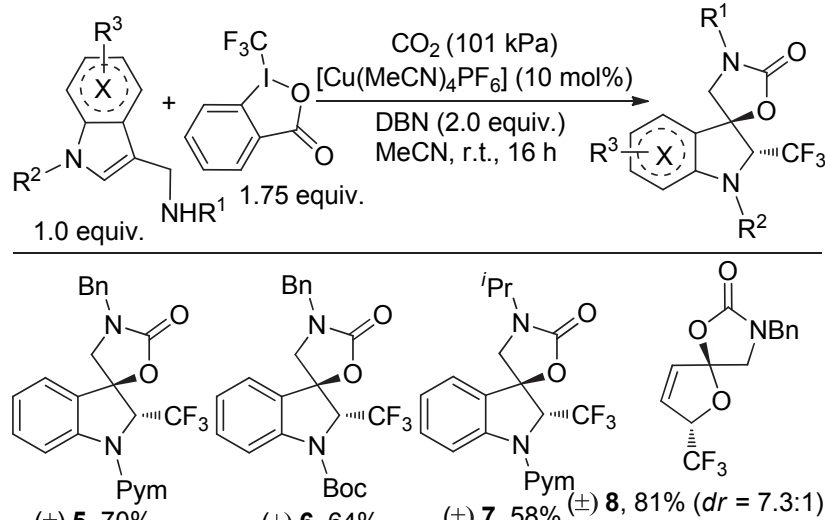

( \pm ) $5,70 \%$

(士) 6, 64\%

(土) $7,58 \%{ }^{( \pm)} 8,81 \%(d r=7.3: 1)$

$71 \%(d r=6.0: 1)$

图 $3 \mathrm{CO}_{2}$ 参与的自由基型去芳构化反应

Figure 3 Radical trifluoromethylative dearomatization of indoles and furans with $\mathrm{CO}_{2}$

去芳构化反应，并以中等到较高的收率及较好的 $d r$ 值 得到重要 C-3 螺环二氢吲哚和不饱和螺环缩醛. 值得强 调的是，对于吲哚甲胺类底物，吲哚氮上不同取代基对 反应有较大影响. 当该取代基为吸电子基团时，反应才 可以较好的进行. 其中, 嘧啶基取代的底物反应性最好. 该反应官能团兼容性较好, 常见的官能团, 如卤素(氟、 氯、溴)、醚、硝基和酯基等, 都能很好的兼容. 此外, 糠 胺类衍生物的反应不仅可以构建双五元环螺缩醛, 还可 以构建五六元环螺缩醛.

\section{1 .2 烯丙胺的氧一全氟烷基化反应}

2017 年，何良年课题组 ${ }^{[20]}$ 使用全氟烷基碘作为自 由基前体，1,5,7-三氮杂二环[4.4.0]癸-5-烯(TBD)作碱, 首次实现了可见光驱动 $\mathrm{CO}_{2}$ 参与的烯丙胺的氧一全氟烷 基化反应，高效构建噁唑啉酮的同时引入重要的全氟烷 基(图 4). 该反应条件温和，无需过渡金属. 全氟烷基

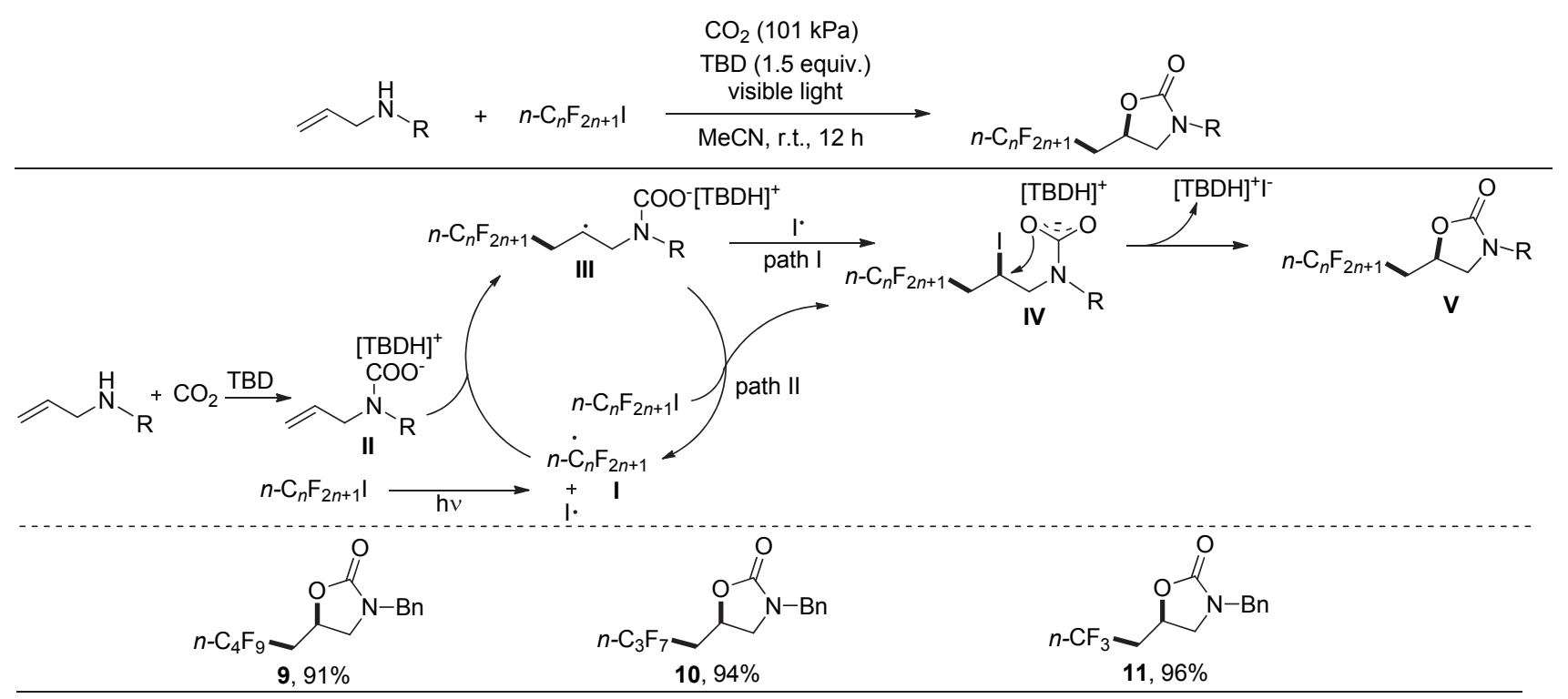

图 $4 \mathrm{CO}_{2}$ 参与的烯丙胺的氧一全氟烷基化反应

Figure 4 Carboxylative cyclization of allyl amines with $\mathrm{CO}_{2}$ 
碘试剂由于活性较高, 可以直接在光照下均裂, 因此无 需加额外的光敏剂. 另外, 该类反应也可以使用烯丙醇 作为底物(图 5), 得到全氟烷基化的环碳酸酯.

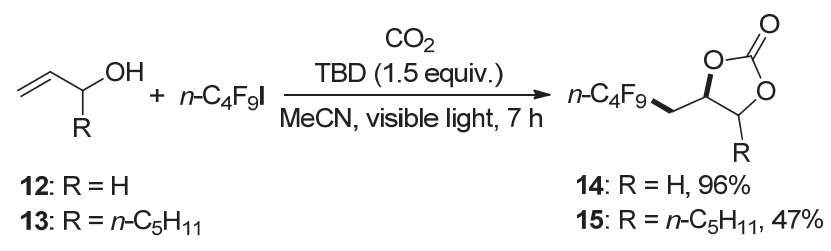

图 $5 \mathrm{CO}_{2}$ 参与的烯丙醇的氧一全氟烷基化反应

Figure 5 Carboxylative cyclization of allyl alcohols with $\mathrm{CO}_{2}$

机理方面，该反应首先通过可见光引发全氟烷基碘 (如 $n-\mathrm{C}_{4} \mathrm{~F}_{9} \mathrm{I}$ )发生 $\mathrm{C}-\mathrm{I}$ 键的均裂, 产生碘自由基和全氟 烷基自由基，随后全氟烷基自由基对原位形成的氨基甲 酸盐中的碳碳双键加成, 得到全氟烷基取代的仲碳自由 基物种 III，该物种随后被碘自由基或全氟烷基碘代物
捕获，形成关键的碘代全氟烷基化氨基甲酸盐中间体 IV. 最后, 经分子内亲核环化得到全氟烷基化的噁唑啉 酮 $\mathbf{V}$.

\section{1 .3 氧一二氟烷基化}

2018 年, 在实现了铜催化烯丙胺的氧一三氟甲基化 反应的基础上, 我们课题组考虑到可见光催化是实现类 似自由基化学的重要方法, 进一步发展了可见光催化 $\mathrm{CO}_{2}$ 参与的烯丙胺的氧-二氟烷基化反应 ${ }^{[21]}$ (图 6), 为各 种重要的二氟烷基取代噁唑啉酮及其衍生物的制备提 供了新途径, 具有良好的官能团兼容性、广泛的底物范 围、可克级规模化、反应条件温和环境友好等特点.

机理研究表明，反应体系中的三乙烯二胺 (DABCO)对激发态的光催化剂 $\mathrm{Ru}(\mathrm{II})$ *进行还原猝灭产 生 $\mathrm{Ru}(\mathrm{I})$. 作者认为, 该 $\mathrm{Ru}(\mathrm{I})$ 可以单电子还原 $\mathrm{BrCF}_{2}$ COOEt 形成二氟烷基自由基. 该自由基与氨基甲酸盐 $\mathbf{I}$ 反应产生苄基自由基 II，随后苄基自由基 II 被激发态的
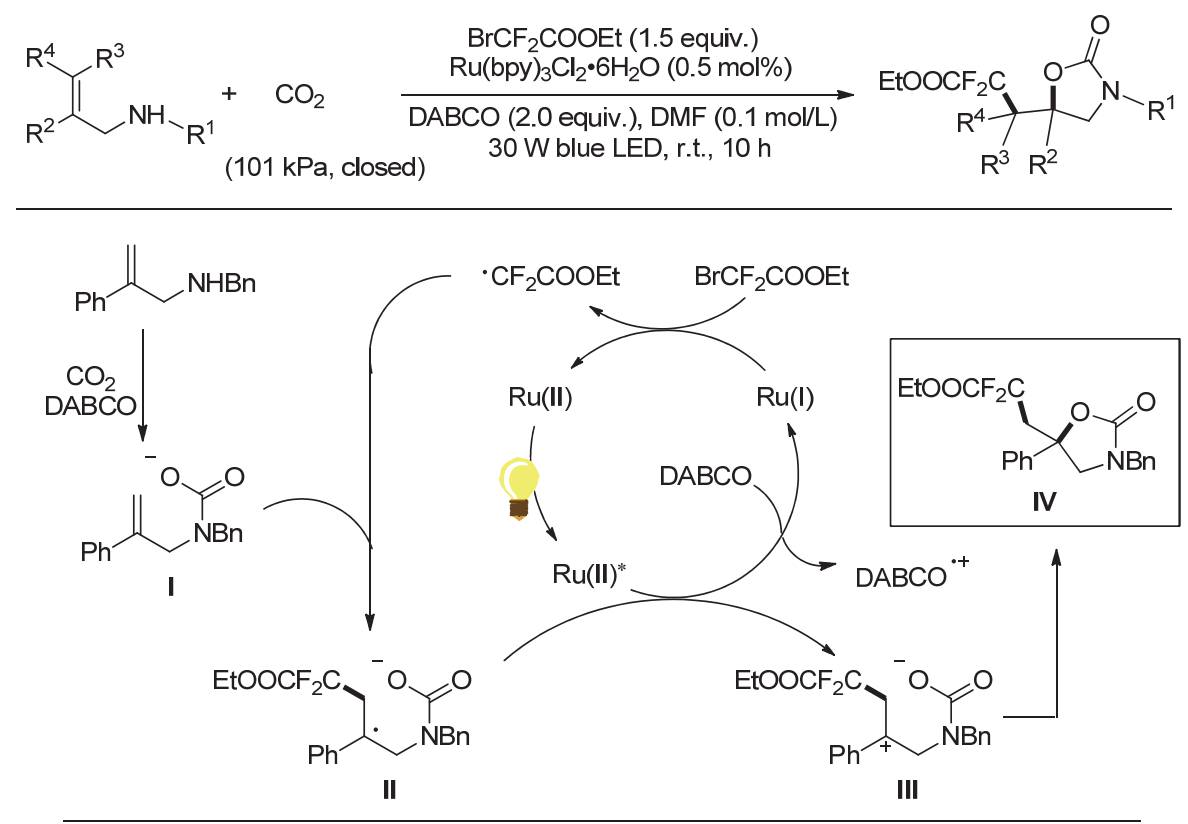

a. Scope of allylamines
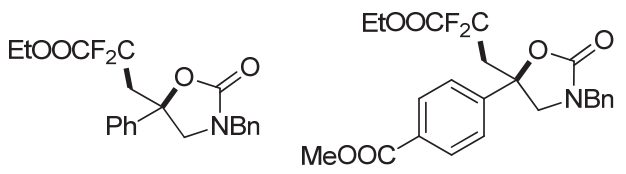

$\mathrm{EtOOCF}_{2} \mathrm{C}$

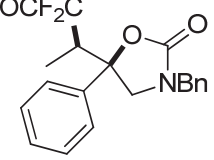

$16,82 \%$

$17,86 \%$

$18,74 \%$

b. Scope of difluoroalkyl reagents

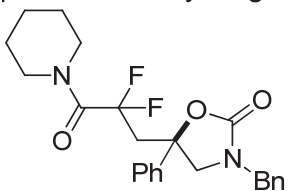

$\mathrm{Et}_{2} \mathrm{NOCF}_{2} \mathrm{C}$<smiles>CCC1(c2ccccc2)CN(Cc2ccccc2)C(=O)O1</smiles>

$(\mathrm{EtO})_{2} \mathrm{OPF}_{2} \mathrm{C}$

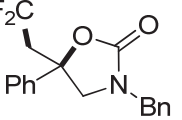

$19,77 \%$

$20,78 \%$

21, $66 \%$

图 $6 \mathrm{CO}_{2}$ 参与的烯丙胺的氧一二氟烷基化

Figure 6 Oxy-difluoroalkylation of allylamines with $\mathrm{CO}_{2}$ 
$\mathrm{Ru}(\mathrm{II})$ 氧化形成苄基正离子 III, 然后进行分子内环化得 到目标产物 IV.

\section{1 .4 氧一烷基化反应}

2017 年, 我们课题组 ${ }^{[22]}$ 报道了可见光驱动钯催化 $\mathrm{C}-\mathrm{H}$ 键与非活化的烷基溴代物的自由基型烷基化反应. 在该反应中, 我们推测活性钯(0)络合物可与叔丁基溴 在光照下发生单电子转移(SET)过程, 产生叔丁基自由 基. 考虑到如前所述的几例自由基型噁唑啉酮合成方法 都需要使用活性较高的亲电试剂, 不能适用于活性较低 的普通烷基卤代物, 我们课题组 ${ }^{[23]}$ 在 2018 年进一步实 现了 $\mathrm{CO}_{2}$ 参与的可见光驱动钯催化的烯丙胺和非活化 烷基溴代物的氧一烷基化反应(图 7). 该三组分反应以 $\operatorname{Pd}\left(\mathrm{PPh}_{3}\right)_{4}$ 为催化剂, 反应条件温和, 底物适用范围广且 产物易于衍生. 反应中各种非活化的烷基溴代物都可以 参与反应, 生成重要的 2-噁唑啉酮类化合物. 总体而言, 一级烷基溴代物的反应性比二、三级烷基溴代物的反应 性差，可能与相应烷基自由基的稳定性有关.

机理研究表明, 该反应历程中有烷基自由基的存 在. 作者认为, 该反应首先通过光激发 $\operatorname{Pd}(0)$ 催化剂引 发催化循环. 激发态的 $\operatorname{Pd}(0)$ 络合物 $\mathbf{I}$ 和烷基溴代物经过 单电子转移产生相应的 $\operatorname{Pd}(\mathrm{I})$ 络合物和烷基自由基 II. 同时, 底物烯丙胺在 $\mathrm{TBD}$ 存在下与 $\mathrm{CO}_{2}$ 原位形成氨基
甲酸盐 III. 随后，烷基自由基 II 与氨基甲酸盐 III 中的 烯烃加成产生更稳定的茮基自由基物种 IV. 接着, $\operatorname{Pd}(\mathrm{I})$ 物种和中间体 IV 经单电子转移生成中间体 $\mathrm{V}$, 同时再 生 $\operatorname{Pd}(0)$ 催化剂(path A), 最后中间体 $\mathbf{V}$ 发生分子内亲核 反应得到所需的啞唑啉酮 VI. 我们认为, 中间体 IV 也 可能经历类似于图 2 所示的自由基-自由基偶联，直接 形成碳氧键并重生 $\operatorname{Pd}(0)$ 催化剂(path B, 图 7).

\section{$2.2 \mathrm{CO}_{2}$ 参与的芳基烯烃 $\alpha$ 羧基化反应}

如前所述, $\mathrm{CO}_{2}$ 参与的芳基烯烃翔基化反应以氢䍨 基化为主, 近年来人们通过自由基化学成功实现了双官 能团化的 $\alpha$ 羧基化反应，主要包括碳羧基化和硅羧基化 两类反应. 从反应机制上讲，主要是自由基前体在光催 化条件下产生自由基，随后进攻烯烃，形成碳自由基， 碳自由基再进一步被还原为碳负离子, 最后进攻 $\mathrm{CO}_{2}$ 生 成取代的 $\alpha$ 羧酸等重要化合物(path B, 图 1).

\subsection{1 碳羧基化反应}

2017 年, Martin 课题组 ${ }^{[24]}$ 报道了可见光促进、 $\mathrm{CO}_{2}$ 参与的芳基烯烃的双官能团化羧基化反应(图 8). 该反 应使用金属 Ir 络合物作为光敏剂, 以 Langlois 试剂(三氟 甲磺酸钠)、二氟甲磺酸钠、苄基三氟硼酸钾盐或草酸 单酯酸盐为自由基前体, 在氧化还原中性条件下实现了 $\mathrm{CO}_{2}$ 参与的芳基烯烃的选择性碳羧基化反应.
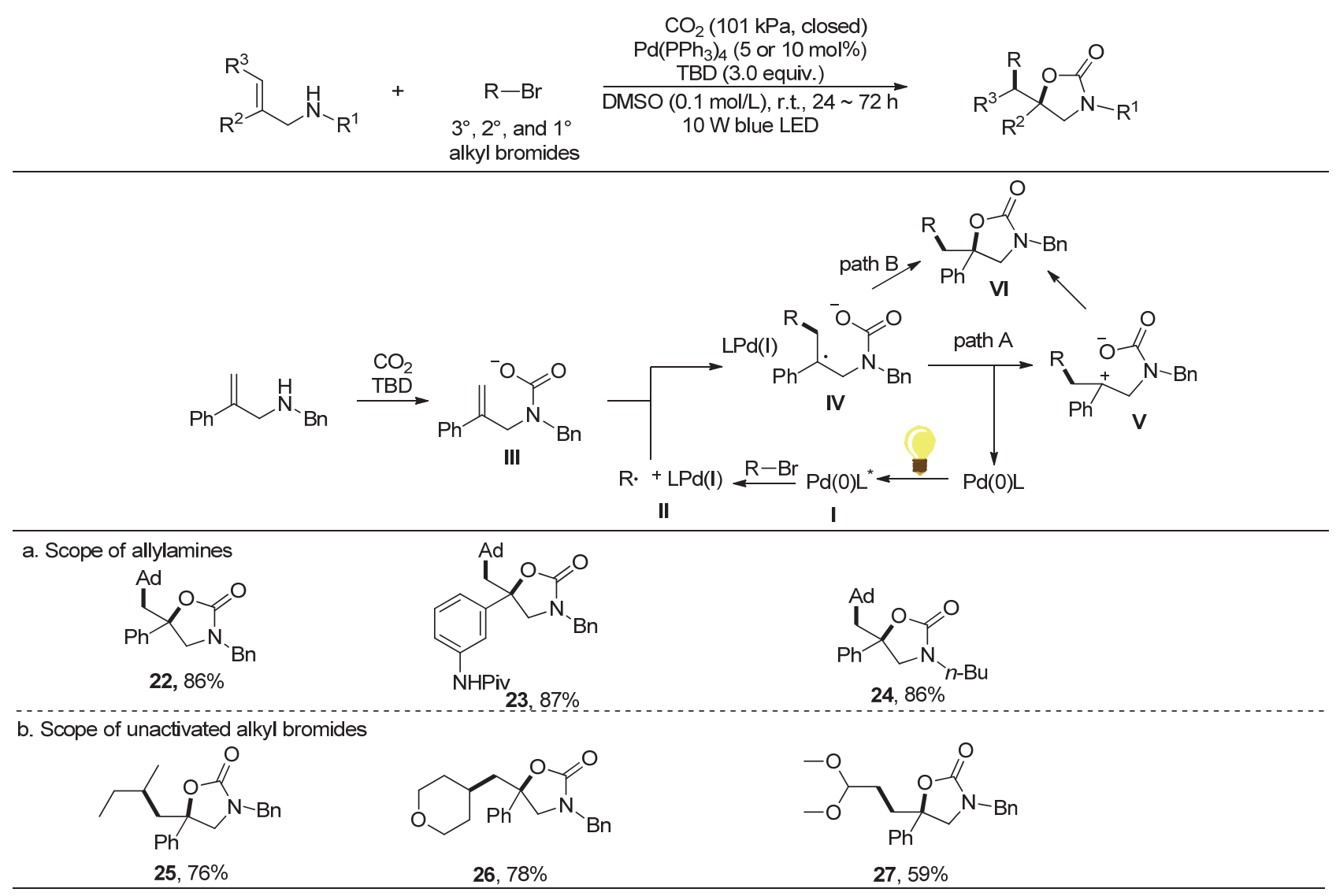

图 7 可见光驱动钯催化的烯丙胺的氧一烷基化反应

Figure 7 Oxy-alkylation of allylamines via visible-light-driven palladium catalysis 

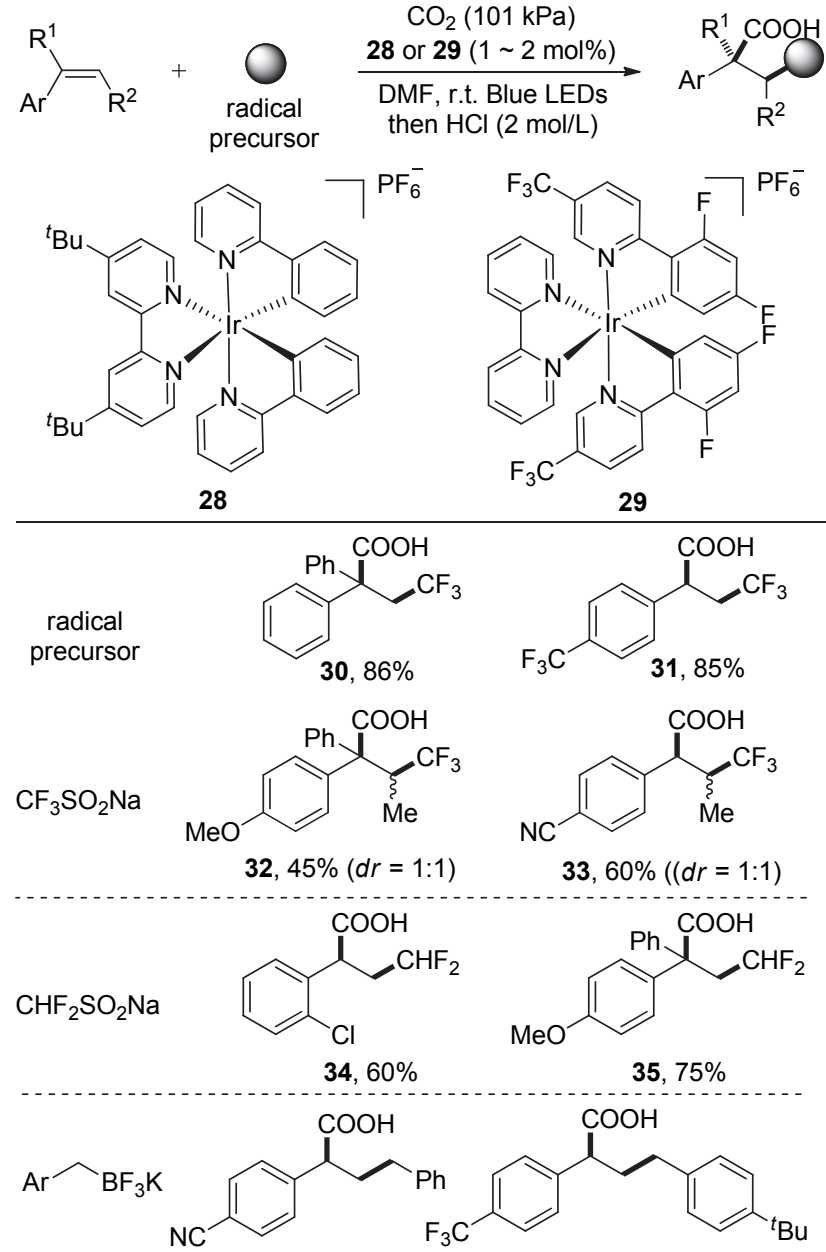

$36,55 \% \quad 37,50 \%$

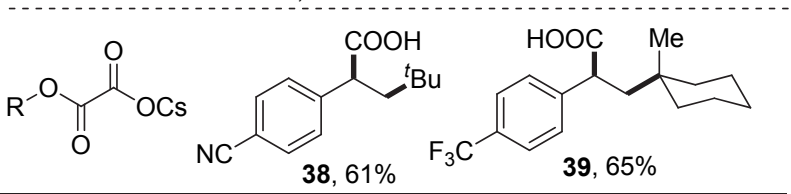

图 $8 \mathrm{CO}_{2}$ 参与的烯烃双官能团化反应

Figure 8 Dicarbofunctionalization of styrenes with $\mathrm{CO}_{2}$

根据机理实验, 作者提出了以下机理(图 9). 激发态 的光敏剂 $\operatorname{Ir}(\mathrm{III}) *$ 通过单电子氧化自由基前体产生碳自 由基物种并生成 $\operatorname{Ir}(\mathrm{II})$, 随后该碳自由基物种对芳基烯 烃加成得到苄基自由基物种(40), 此物种被还原态的光 敏剂 $\operatorname{Ir}(\mathrm{II})$ 单电子还原为苄基碳负离子(41) 并与 $\mathrm{CO}_{2}$ 反应 得到羧基化产物. 整个反应的条件简单温和, 不需要额 外加入有机金属还原剂、空气敏感的有机金属试剂及特 殊的配体, 为实现碳羧基化反应提供了新的策略. 此外, 该反应可引入重要的含氟砌块和多种烷基, 具有广阔的 应用前景. 但反应底物主要为芳基烯烃等活化烯烃, 体 系并不适用一般非活化的烯烃.

\subsection{2 硅羒基化反应}

2018 年, 吴杰课题组 ${ }^{[25}$ (图 10)利用光催化剂和氢原 子转移催化剂的协同作用, 首次实现了光催化 $\mathrm{CO}_{2}$ 参与

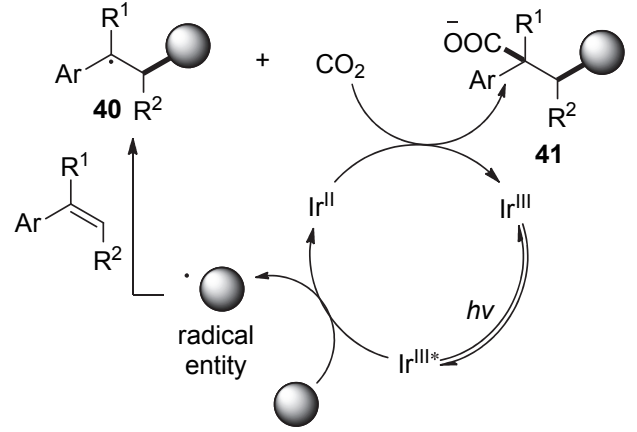

图 $9 \mathrm{CO}_{2}$ 参与的芳基烯烃碳羧基化反应机理

Figure 9 A proposed mechanism of dicarbofunctionalization of styrenes with $\mathrm{CO}_{2}$ and radical precursors

的烯烃硅羧基化反应. 此外，该策略也可以胺类或者醚 类为原料，通过碳氢键直接官能团化实现烯烃的碳羧基 化反应. 反应以 4CzIPN 作为光催化剂, 3-乙酰氧基奎宁 为氢原子转移催化剂, 在室温和蓝光照射下, 一系列取 代苯乙烯与硅烷在 $101 \mathrm{kPa}$ 的 $\mathrm{CO}_{2}$ 氛围下反应, 以较高 的收率生成烯烃硅羧基化产物. 在用叔丁氧羰基(Boc) 保护的四氢吡咯作为自由基前体时，该策略同样可以实 现烯烃的碳羧基化反应，并得到重要的 $\gamma$-氨基酸类产 物。该反应在氧化还原中性条件下通过硅氢键活化或碳 氢键活化实现烯烃的双官能团化，反应无需外加氧化 剂，具有很好的原子经济性. 此外，该反应条件温和， 可利用连续流技术实现克级反应. 该反应除了贫电子的 芳基烯烃，还适用于丙烯酸酯、丙烯酰胺、烯基砜、丙 烯腈等贫电子烯烃，但对于非活化的普通烯烃并不兼 容.

机理研究表明(图 11), 激发态的 4CzIPN*首先被 3乙酰氧基奎宁还原淬灭得到 4CzIPN 自由基负离子 $\mathrm{A}$, 同时生成自由基阳离子中间体 $\mathrm{B}$; 随后，自由基阳离子 $\mathrm{B}$ 促进硅氢键或碳氢键的氢原子转移产生硅或碳自由基 $\mathrm{D}$, 同时产生奎宁阳离子 $\mathrm{C}$, 硅或碳自由基 $\mathrm{D}$ 与烯烃加 成形成碳自由基物种 $\mathrm{E}$; 接着, 碳自由基物种 $\mathrm{E}$ 被还原 态的 4CzIPN (A)还原得到碳负离子中间体 F, 随后亲核 进攻 $\mathrm{CO}_{2}$ 得到 $\beta$-硅基羧酸或含有 $\gamma$-杂原子的羧酸产物.

\section{$2.3 \mathrm{CO}_{2}$ 参与的 $\beta$ 羧基化反应}

相较于 $\alpha$-羧基化反应，通过烯烃双官能团化生成 $\beta$ 羧基化产物, 无论从 $\mathrm{CO}_{2}$ 的活化模式还是从反应位点选 择性来讲, 都更具有挑战性. 目前实现该类反应的例子 非常少.

\subsection{1 硫羧基化反应}

2017 年, 我们课题组 ${ }^{[26]}$ 首次报道了可见光驱动铁 促进 $\mathrm{CO}_{2}$ 参与的苯乙烯和丙烯酸酯的硫羧基化反应(图 12). 反应以叔丁醇钾作碱, 氯化铁为催化剂, 在室温条 件下，不同种类的苯乙烯和丙烯酸酯都可以很好地反 应, 并以非常高的收率得到重要的 $\beta$-硫代羧酸产物. 

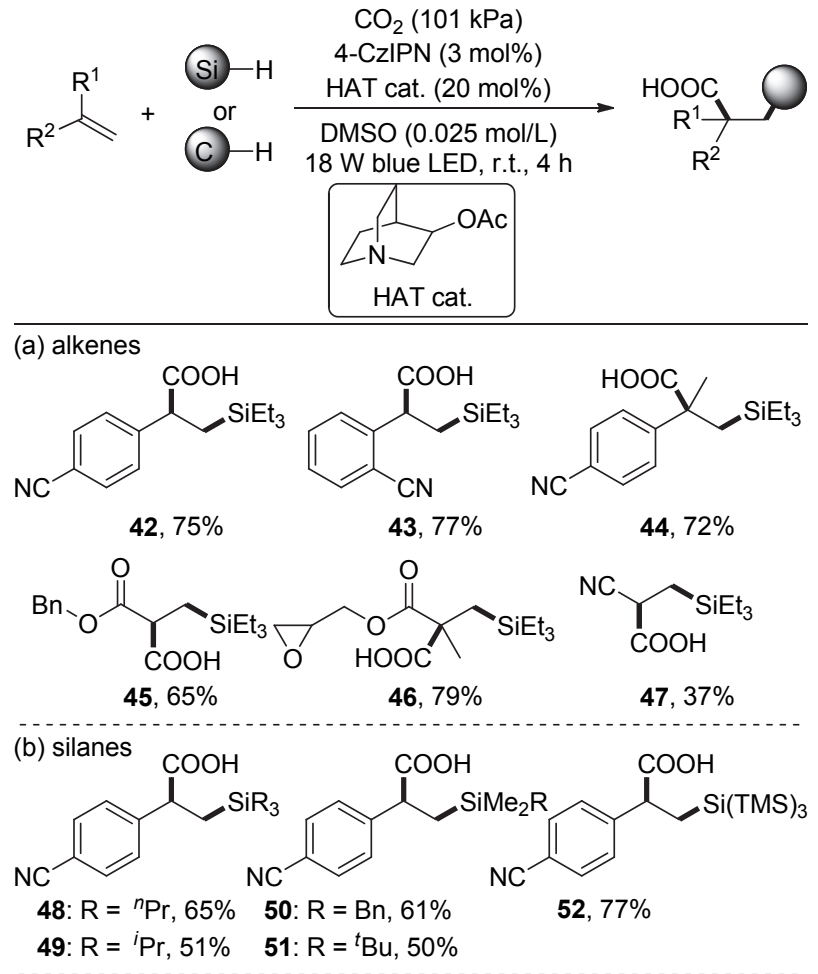

(c) alkanes<smiles>CC(C)(CC1CCCN1C(=O)OCc1ccccc1)c1ccc(C#N)cc1</smiles>

53, $58 \%(d r=1: 1.6)$

$\mathbf{5 4}, 60 \%(d r=1: 1)$

55, $59 \%(d r=1: 1)$<smiles>CN(CCC(C(=O)O)c1ccc(C#N)cc1)C(=O)C#N</smiles>

56, $65 \%(d r=1: 1)$

$57,65 \%$

$58,50 \%$

图 $10 \mathrm{CO}_{2}$ 参与的烯烃与硅烷或烷烃的双官能团化反应

Figure 10 Dicarbofunctionalization of alkenes with $\mathrm{CO}_{2}$ and silanes or alkanes

值得一提的是, 与以往报道的烯烃 $\alpha$-选择性羧基化 反应不同，该反应的羧基化发生在苯乙烯的 $\beta$ 位，具有 很好的区域选择性. 除此之外，该反应条件温和，具有
高的化学选择性和非对映选择性, 生成的产物易于转化 为生物活性物种和重要药物分子.

经过自由基捕获实验和自由基钟实验，我们推测该 反应可能经历自由基历程. 虽然目前还缺乏证据我们认 为该反应的可能机理如图 13 所示. 首先, 叔丁醇钾作为 碱实现硫酚的去质子，并促进硫铁复合物的形成，硫铁 复合物作为光致氧化还原催化剂或电子转移促进剂. 在 可见光照射下，激发态硫铁复合物与 $\mathrm{CO}_{2}$ 发生单电子转 移形成 $\mathrm{CO}_{2}$ 自由基负离子和氧化态的硫铁复合物, 而后 $\mathrm{CO}_{2}$ 自由基负离子与苯乙烯加成得到稳定的茮基自由基 物种, 最后与氧化态硫铁复合物反应得到 $\beta$-硫代羧酸产 物，并重生活性硫铁复合物.

该反应提供了新型的二氧化碳活化模式, 为后续二 氧化碳的活化和利用提供了很好的借鉴，但目前实现的 硫羧基化反应，底物范围还具有一定的局限性，比如底 物之一的烯烃, 目前只适用于活化烯烃(包括苯乙烯类 化合物、丙烯酸酯类化合物); 硫源中，供电子性的硫酚 化合物反应较好，贫电子硫酚以及普通硫醇在该反应中 不兼容.

\section{$2.4 \mathrm{CO}_{2}$ 参与的烯烃双羧基化反应}

除通过光催化实现烯烃双官能团化反应外，电催化 烯烃双官能团化反应也有相关报道，较为常见的反应类 型为烯烃的双羧基化反应，可以构建在药物、材料等工 业中有着非常重要作用的丁二酸类衍生物. 早在 2001 年, Senboku 和 Tokuda 组 ${ }^{[27]}$ 就报道了 $\mathrm{CO}_{2}$ 参与的苯基丁 二酸的合成(图 14). 反应中, 在镁为阳极、铂为阴极的 条件下, 苯基取代的烯烃与二氧化碳高效的反应并以中 等或较好的收率得到目标产物苯基丁二酸. 反应中，作 者提出了两条可能的路径: 路径 $\mathrm{a}$, 由于苯乙烯及其衍 生物相较于二氧化碳有更低的还原电势, 所以 $\mathrm{CO}_{2}$ 在反 应体系中被还原为 $\mathrm{CO}_{2}$ 自由基负离子后进攻烯烃，形成 自由基中间体 $\mathrm{A}$, 中间体 $\mathrm{A}$ 进一步被还原为碳负离子并 直接与另一分子 $\mathrm{CO}_{2}$ 反应形成二酸. 路径 $\mathrm{b}$, 对于 $1,2-$ 二苯基乙烯类化合物，由于还原电势类似于 $\mathrm{CO}_{2}$, 烯烃 类化合物可被还原为烯烃自由基负离子，进而与 $\mathrm{CO}_{2}$ 反 应生成类似 $\mathrm{A}$ 的中间体 $\mathrm{B}$ ，中间体 $\mathrm{B}$ 进一步被还原为碳 负离子并进一步与 $\mathrm{CO}_{2}$ 反应生成二酸(图 1, path D).

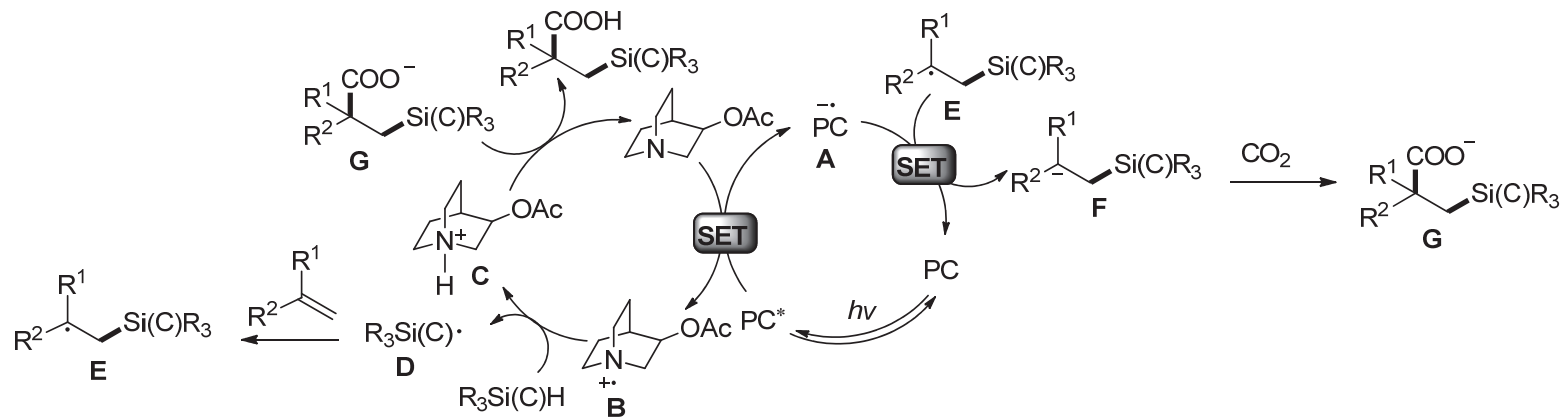

图 $11 \mathrm{CO}_{2}$ 参与的烯烃与硅烷或烷烃的双官能团化反应机理

Figure 11 A proposed mechanism of dicarbofunctionalization of alkenes with $\mathrm{CO}_{2}$ and silanes or alkanes 

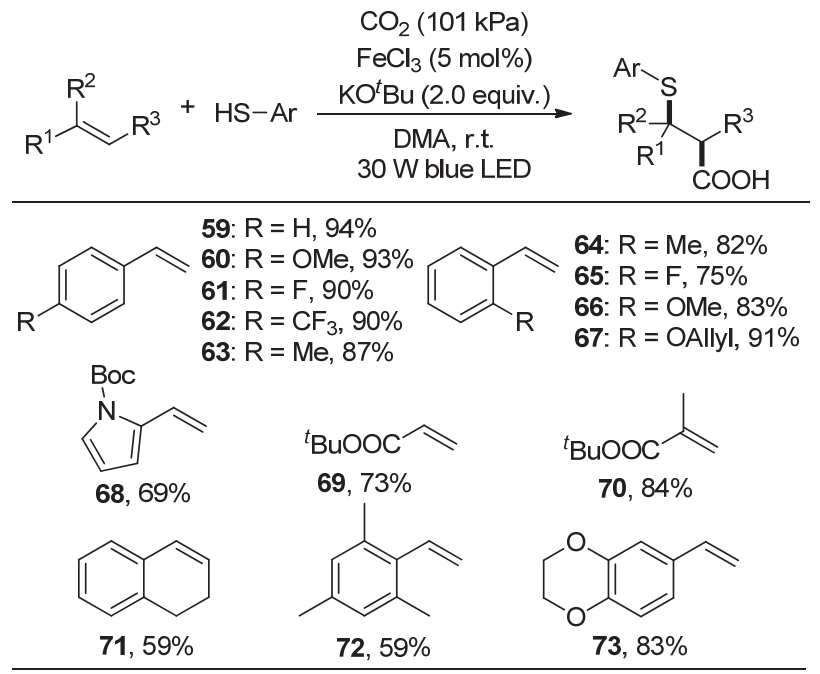

图 12 可见光驱动铁促进 $\mathrm{CO}_{2}$ 参与的烯烃与苯硫酚的硫羧基化反应 Figure 12 Visible-light-driven iron-promoted thiocarboxylation of alkenes with $\mathrm{CO}_{2}$

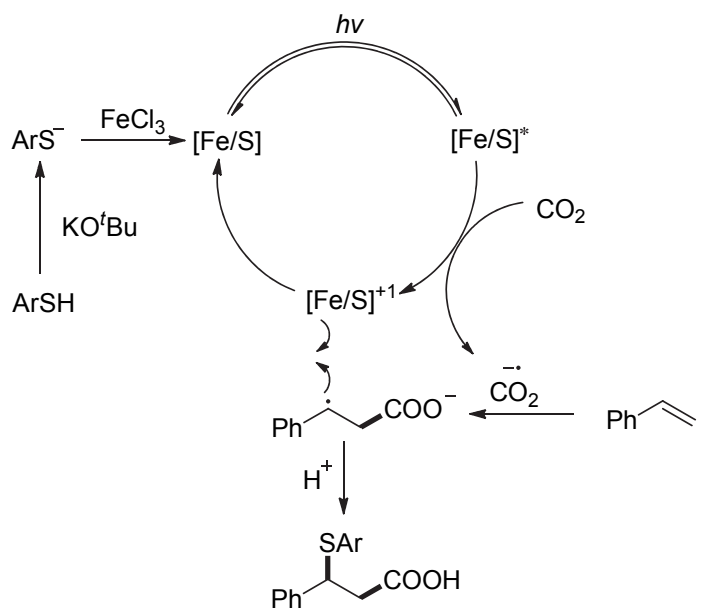

图 13 可见光驱动铁促进 $\mathrm{CO}_{2}$ 参与的烯烃与苯硫酚的硫羧基化反应 机理

Figure 13 A proposed mechanism of visible-light-driven iron-promoted thiocarboxylation of alkenes with $\mathrm{CO}_{2}$

随后江焕峰课题组用非贵金属 $\mathrm{Ni}$ 代替 Pt 作为阴极, $\mathrm{Al}$ 作为牺牲阳极, 高效地实现了芳基取代的烯烃 ${ }^{[2]}$ 和 1,3-丁二烯 ${ }^{[29]}$ 的双羧基化反应(图 15), 分别构建重要的 丁二酸和已二酸类衍生物, 具有很重要合成意义. 该类 反应收率良好, 对于 1,3-丁二烯类化合物几乎可以单一 的 $E$ 式构型得到目标产物(1,3-丁二烯除外, 得到的是 $1: 1$ 的混合物), 阴极电极除了 $\mathrm{Ni}$ 电极, $\mathrm{Cu}$ 电极也有不 错的效果.

\section{3 总结与展望}

虽然 $\mathrm{CO}_{2}$ 参与的自由基型烯烃双官能团化反应已 经取得一些重要进展, 为相关领域的发展提供了很好的 借鉴，但是该领域目前还处于发展阶段，仍然面临众多
(+) $\mathrm{Mg}(-) \mathrm{Pt}$

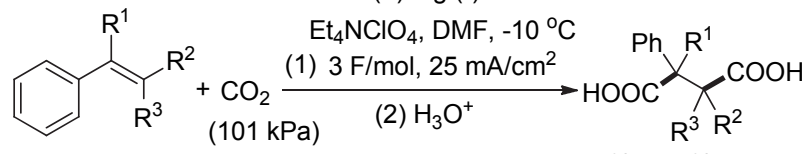
$65 \% \sim 91 \%$ yields

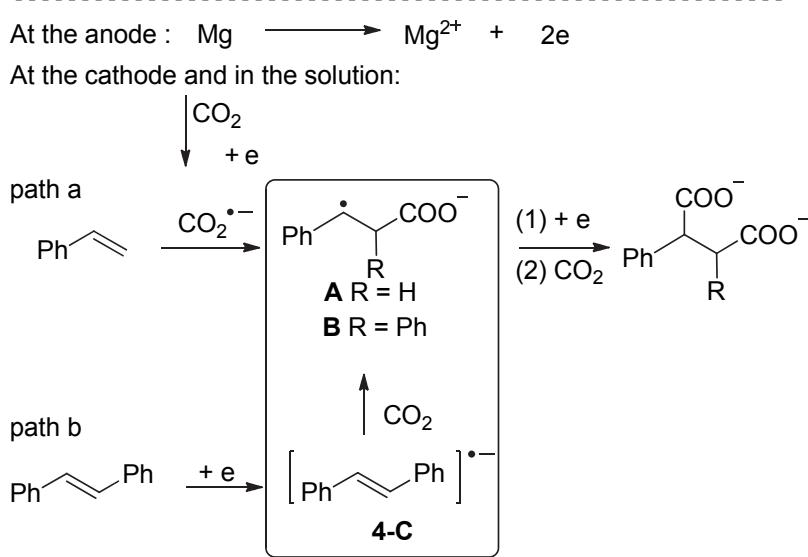

图 14 Senboku 和 Tokuda 组电催化 $\mathrm{CO}_{2}$ 参与的烯烃双羧基化反应 Figure 14 Electrosynthesis of 2-phenylsuccinic acids with $\mathrm{CO}_{2}$ by Senboku and Tokuda group

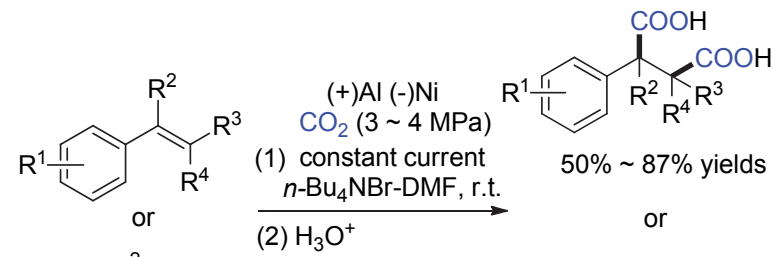<smiles>[R]C=C([R])C([R])=C[R]</smiles><smiles>[R]C(C(=O)O)C([R])C([R])C(=O)O</smiles>

$70 \% \sim 90 \%$ yields only $E$ form (except for 1,3-butadiene, $Z: E=1: 1$ )

图 15 江焕峰课题组电催化 $\mathrm{CO}_{2}$ 参与烯烃和 1,3-丁二烯的双羧基化反 应

Figure 15 Electrochemical dicarboxylation of arylalkenes and 1,3-dienes with $\mathrm{CO}_{2}$ by Jiang group

挑战，需要进一步发展，主要表现在以下几个方面.

一、 $\mathrm{CO}_{2}$ 参与自由基型反应构建噁唑啉酮类杂环化 合物的方法存在以下不足: (a)已合成的噁唑啉酮类化合 物的取代类型还局限于引入烷基, 还需进一步利用杂原 子自由基参与类似转化, 合成具有杂原子的噁唑啉酮; (b)此类反应一般基于(path A, 图 1)这样的路径, 只实现 了分子内关环反应，目前还未实现更具挑战性的分子间 反应；(c)该类反应的立体选择性还不能很好控制，需要 进一步深入研究. 此外, 该类方法应该可以拓展至杂原 子自由基前体参与的反应以及其他重要含羰基杂环(如 环碳酸酯)的高效合成中.

二、通过烯烃自由基型双官能团化反应实现烯烃的 碳羧基化、硅羧基化和硫羧基化等羧基化反应，虽然反 应机制不尽相同，但都能够在温和的反应条件下实现较 好的选择性和较高的收率, 具有较好的潜在工业化应用 
前景. 该类转化目前还有以下几个问题需进一步解决: (a)底物普适性有待进一步提高. 目前底物范围还比较 局限，主要集中在芳基烯烃、丙烯酸酯等这类活化烯烃， 而对于非活化的普通烯烃还不能实现其双官能团化, 可 能原因是反应过程中非活化烯烃产生的自由基中间体 稳定性较差, 也难于进一步还原为碳负离子, 即使生成 的羧酸盐也容易发生脱羧, 不利于催化反应进行; (b)产 物类型存在一定局限. 目前只实现了碳羧基化、硫羧基 化、硅羧基化、膦羧基化 ${ }^{[30]}$ 反应等少数几个类型, 而其 他类型, 如自由基型氮羧基化等非常重要的反应目前还 未见报道; (c)从选择性来看, 目前虽然实现特定区域选 择性, 但还不能通过条件简单变化实现两种区域选择性 的调控; 此外, 对映选择性的控制目前还非常困难, 如 何发展新型不对称催化体系实现手性羧酸的合成具有 重大挑战. 除此而外, 相关反应机理的研究目前尚不够 深入, 实验数据尚不能完全说明提出的机理, 所以相关 反应机理研究也将是该领域未来发展的重点.

作者相信, 随着自由基化学的快速发展、二氧化碳 活化模式以及烯烃双官能团化催化体系的不断创新，化 学家们一定可以实现更多更有价值的二氧化碳参与的 自由基型烯烃双官能团化反应, 变废为宝, 将二氧化碳 化学转化变得真正有用, 从而实现工业化, 为相关精细 化学品的合成、以及资源和能源问题的解决提供新的思 路.

\section{作者简介}

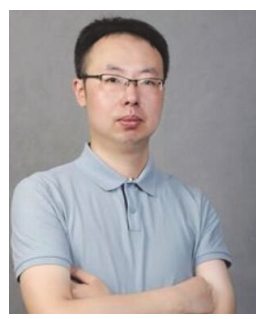

张振, 特聘副研究员, 1985 年出生于山西怀仁市, 2007 年 在四川大学化学学院获得学士学位, 2017 年在中科院成都有 机所获得博士学位(导师: 支永刚研究员和余达刚教授), 随后 在成都大学药学与生物工程学院工作至今. 其研究兴趣主要 是药物合成、二氧化碳化学.

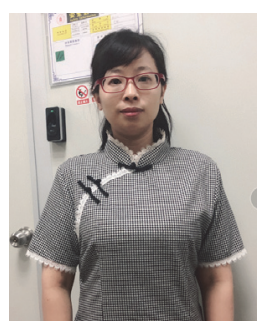

李静, 助理研究员, 1981 年出生于四川广安市, 2006 年在 四川大学化学学院获得学士学位, 2009 年在四川大学化学学 院获得硕士学位(导师: 余孝其教授), 随后在四川抗菌素工业 研究所工作至 2012 年, 2012 年 6 月在四川大学化学学院工作
至今. 其研究兴趣主要是二氧化碳化学、无机-有机杂化材料 的合成.

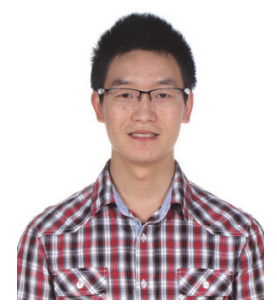

余达刚, 2007 年本科毕业于四川大学化学学院, 2012 年博 士毕业于北京大学化学与分子工程学院(导师: 施章杰教授), 2012 2014 年作为洪堡学者在德国明斯特大学(导师：Frank Glorius 教授)开展博士后研究, 2015 年回到四川大学开展教学 和独立研究工作, 被聘为教授、博士生导师. 主要从事二氧化 碳利用和可见光催化等绿色可持续发展化学研究, 已发表学 术论文 60 余篇, 参与管写英文专著 2 个章节, 受邀在国内外 会议上做邀请报告和主题报告 10 余次. 曾入选 2015 年中组部 “青年千人”、四川省“青年千人”和 2016 年“Organic Chemistry Frontiers” Emerging Investigators; 主持了 2018 年国家自然科 学基金委优秀青年基金和霍英东青年教师基金等多项科研项 目; 曾获得 2017 年 Thieme Chemistry Journal Award、2017 年 ACP Lectureship Awards、2018 年中国化学会青年化学奖、2018 年四川大学 “优秀教师” 等奖励.

\section{References}

[1] (a) Aresta, M. Carbon Dioxide as Chemical Feedstock, Wiley-VCH, Weinheim, 2010. (b) He, L.-N. Carbon Dioxide Chemistry, Science Press, Beijing, 2013 (in Chinese). (何良年, 二氧化碳化学, 科学出 版社, 北京, 2013). (c) Centi, G.; Perathoner, S. Green Carbon Dioxide: Advances in $\mathrm{CO}_{2}$ Utilization, Wiley-VCH, Weinheim, 2014.

[2] Selected reviews on $\mathrm{CO}_{2}$ utilization to generate the $\mathrm{C}-\mathrm{O} / \mathrm{C}-\mathrm{C}$ bonds, see: (a) Huang, K.; Sun, C.-L.; Shi, Z.-J. Chem. Soc. Rev. 2011, 40, 2435. (b) Martin, R.; Kleij, A. W. ChemSusChem 2011, 4 1259. (c) Tsuji, Y.; Fujihara, T. Chem. Commun. 2012, 48, 9956. (d) He, M.; Sun, Y.; Han, B. Angew. Chem., Int. Ed. 2013, 52, 9620. (e) Zhang, L.; Hou, Z. Chem. Sci. 2013, 4, 3395. (f) Liu, Q.; Wu, L.; Jackstell, R.; Beller, M. Nat. Commun. 2015, 6, 5933. (g) Börjesson, M.; Moragas, T.; Gallego, D.; Martin, R. ACS Catal. 2016, 6, 6739. (h) Zhang, L.; Han, Z.; Zhang, L.; Li, M.; Ding, K. Chin. J. Org. Chem. 2016, 36, 1824 (in Chinese). (张琳莉, 韩召斌, 张砧, 李明 星, 丁奎岭, 有机化学, 2016, 36, 1824) (i) Zhu, Q.; Wang, L.; Xia, C.; Liu, C. Chin. J. Org. Chem. 2016, 36, 2813 (in Chinese). (朱庆, 王露, 夏春谷, 刘超, 有机化学, 2016, 36, 2813) (j) Zhang, W.; Guo, C.; Lu, X. Chin. J. Catal. 2016, 37, 215. (k) Zhang, H.; Sun, H.; Li, X. Chin. J. Org. Chem. 2016, 36, 2843 (in Chinese). (伅花, 孙宏建, 李晓燕, 有机化学, 2016, 36, 2843.) (1) Zhang, S.; Li, X.; He, L.-N. Acta Chim. Sinica 2016, 74, 17 (in Chinese). (张帅, 李雪 冬, 何良年, 化学学报, Acta Chim. Sinica 2016, 74, 17. (m) Song, Q.-W.; Zhou, Z.-H.; He, L.-N. Green Chem. 2017, 19, 3707. (n) Gui, Y.-Y.; Zhou, W.-J.; Ye, J.-H.; Yu, D.-G. ChemSusChem 2017, 10, 1337. (o) Luo, J.; Larrosa, I. ChemSusChem 2017, 10, 3317. (p) Zhang, Z.; Ju, T.; Ye, J.-H.; Yu, D.-G. Synlett 2017, 28, 741. (q) Zou, B.; Hu, C. Chin. J. Chem. 2017, 35, 541. (r) Li, Y.; Wang, Z.; Liu, Q. Chin. J. Org. Chem. 2017, 37, 1978 (in Chinese). (李勇, 王征, 刘 庆涁, 有机化学, 2017, 37, 1978) (s) Zhang, W.; Zhang, N.; Guo, C.; Lü, X. Chin. J. Org. Chem. 2017, 37, 1309 (in Chinese). (张文 珍, 张宁, 郭春晓, 吕小兵, 有机化学, 2017, 37, 1309) (t) Feng, J.; Zeng, S.; Feng, J.; Dong, H.; Zhang, X. Chin. J. Chem. 2018, 36, 961. (u) Zhao, Y.; Liu, Z. Chin. J. Chem. 2018, 36, 455; (v) Zhang, Y.; Cen, J.; Xiong, W.; Qi, C.; Jiang, H. Prog. Chem. 2018, 30, 547 (in Chinese). (张宇, 岑竞鹤, 熊文芳, 戚朝荣, 江焕峰, 化学进 展, 2018, 30, 547.) (w) Wang, L.; Sun, W.; Liu, C. Chin. J. Chem. 2018, 36, 353. (x) Chen, Y.-G.; Xu, X.-T.; Zhang, K.; Li, Y.-Q.; Zhang, L.-P.; Fang, P.; Mei, T.-S. Synthesis 2018, 50, 35. (y) Wang, 
S.; Xi, C. Chem. Soc. Rev. 2019, 48, 382. (z) Chen, Z.; Liu, J.; Cui, H.; Zhang, L.; Su, C. Acta Chim. Sinica 2019, 77, 242 (in Chinese). (陈之尧, 刘捷威, 崔浩, 张利, 苏成勇, 化学学报, 2019, 77, 242.)

[3] (a) Sasano, K.; Takaya, J.; Iwasawa, N. J. Am. Chem. Soc. 2013, 135, 1251. (b) Sekine, K.; Sadamitsu, Y.; Yamada, T. Org. Lett. 2015, 17, 5706. (c) Moragas, T.; Gaydou, M.; Martin, R. Angew. Chem., Int. Ed. 2016, 55, 5053. (d) Miao, B.; Li, S.; Li, G.; Ma, S. Org. Lett. 2016, 18, 2556. (e) Nogi, K.; Fujihara, T.; Terao, J.; Tsuji, Y. J. Am. Chem. Soc. 2016, 138, 5547. (f) Gholap, S. S.; Takimoto, M.; Hou, Z. Chem. Eur. J. 2016, 22, 8547. (g) Yan, S.-S.; Zhu, L.; Ye, J.-H.; Zhang, Z.; Huang, H.; Zeng, H.; Li, C.-J.; Lan, Y.; Yu, D.-G. Chem. Sci. 2018, 9, 4873. (h) Song, L.; Zhu, L.; Zhang, Z.; Ye, J.-H.; Yan, S.-S.; Han, J.-L.; Yin, Z.-B.; Lan, Y.; Yu, D.-G. Org. Lett. 2018, 20, 3776. (i) Fu, L.; Li, S.; Cai, Z.; Ding, Y.; Guo, X.; Zhou, L.; Yuan, D.; Sun, Q.; Li, G. Nat. Catal. 2018, 1, 469. (j) Xiong, W. F.; Yan, D. H.; Qi, C. R.; Jiang, H. F. Org. Lett. 2018, 20, 672. (k) Wang, S.; Xi, C. J. Org. Lett. 2018, 20, 4131. (1) Song, L.; Cao, G.-M.; Zhou, W.; Ye, J.-H.; Zhang, Z.; Tian, X.-Y.; Li, J.; Yu, D.-G. Org. Chem. Front. 2018, 5, 2086. (m) Cai, Z.; Li, S.; Gao, Y.; Li, G. Adv. Synth. Catal. 2018, 360, 4005. (n) Huang, R.; Li, S.; Fu, L.; Li, G. Asian J. Org. Chem. 2018, 7, 1376. (o) Gao, Y.; Cai, Z.; Li, S.; Li, G. Org. Lett. 2019, 21, 3663. (p) Yan, S.-S.; Wu, D.-S.; Ye, J.-H.; Gong, L.; Zeng, X.; Ran, C.-K.; Gui, Y.-Y.; Li, J.; Yu, D.-G. $A C S$ Catal. 2019, 9, 6987.

[4] (a) Seo, H; Katcher, M. H.; Jamison, T. F. Nat. Chem. 2017, 9, 453. (b) Meng, Q.; Wang, S.; König, B. Angew. Chem., Int. Ed. 2017, 56, 13426. (c) Shimomaki, K.; Murata, K.; Martin, R.; Iwasawa, N. J. Am. Chem. Soc. 2017, 139, 9467. (d) Liao, L.-L.; Cao, G.-M.; Ye, J.-H.; Sun, G.-Q.; Zhou, W.-J.; Gui, Y.-Y.; Yan, S.-S.; Shen, G.; Yu, D.-G. J. Am. Chem. Soc. 2018, 140, 17338. (e) Ju, T.; Fu, Q.; Ye, J.-H.; Zhang, Z.; Liao, L.-L.; Yan, S.-S.; Tian, X.-Y.; Luo, S.-P.; Li, J.; Yu, D.-G. Angew. Chem. Int. Ed. 2018, 57, 13897. (f) Fan, X.; Gong, X.; Ma, M.; Wang, R.; Walsh, P. J. Nat. Commun. 2018, 9, 4936.

[5] (a) Wang, H.; Lin, M.-Y.; Fang, H. J.; Chen, T. T.; Lu, J.-X. Chin. J. Chem. 2007, 25, 913. (b) Wang, H.; Du, Y. F.; Lin, M. Y.; Zhang, K.; Lu, J.-X. Chin. J. Chem. 2008, 26, 1745. (c) Jiao, K.; Li, Z.; Xu, X.; Zhang, L.; Li, Y.; Zhang, K.; Mei, T.-S. Org. Chem. Front. 2018, 5, 2244.

[6] (a) Xin, Z.; Lescot, C.; Friis, S. D.; Daasbjerg, Kim; Skrydstrup, T. Angew. Chem. Int. Ed. 2015, 54, 6862. (b) Zhang, W.; Yang, M. W.; Lv, X. Green Chem. 2016, 18, 4181. (c) Zhang, Z.; Liao, L.-L.; Yan, S.-S.; Wang, L.; He, Y.-Q.; Ye, J.-H.; Li, J.; Zhi, Y.-G.; Yu, D.-G. Angew. Chem., Int. Ed., 2016, 55, 7068. (d) Wang, S.; Shao, P.; Du, G.; Xi, C. J. Org. Chem. 2016, 81, 6672.

[7] (a) Hu, J.; Ma, J.; Zhu, Q.; Zhang, Z.; Wu, C.; Han, B. Angew. Chem. Int. Ed. 2015, 54, 5399. (b) Gao, X.; Yu, B.; Yang, Z.; Zhao, Y.; Zhang, H.; Hao, L.; Han, B.; Liu, Z. ACS Catal. 2015, 5, 6648. (c) Zhao, Y.; Wu, Y.; Yuan, G.; Hao, L.; Gao, X.; Yang, Z.; Yu, B.; Zhang, H.; Liu, Z. Chem. Asian J. 2016, 11, 2735.

[8] (a) Li, Y.; Fang, X.; Junge, K.; Beller, M. Angew. Chem. Int. Ed. 2013, 52, 9568. (b) Zhang, Z.; Sun, Q.; Xia, C.; Sun, W. Org. Lett. 2016, 18, 6316. (c) Zhang, Y.; Wang, H.; Yuan, H.; Shi, F. ACS Sustainable Chem. Eng. 2017, 5, 5758. (d) Ren, X.; Zheng, Z.; Zhang, L.; Wang, Z.; Xia, C.; Ding, K. Angew. Chem., Int. Ed. 2017, 56, 310 .

[9] (a) Lehn, J.-M.; Ziessel, R. Proc. Natl. Acad. Sci. USA 1982, 79, 701. (b) Burgess, S. A.; Kendall, A. J.; Tyler, D. R.; Linehan, J. C.; Appel, A. M. ACS Catal. 2017, 7, 3089.

[10] (a) Pupo, G.; Properzi, R.; List, B. Angew. Chem., Int. Ed. 2016, 55, 6099. (b) Riemer, D.; Mandaviya, B.; Schilling, W.; Götz, A. C.; Kühl, T.; Finger, M.; Das, S. ACS Catal. 2018, 8, 3030. (c) Roy, T.; Kim, M. J.; Yang, Y.; Kim, S.; Kang, G.; Ren, X.; Kadziola, A.; Lee, H.-Y.; Baik, M.-H. Lee, J.-W. ACS Catal. 2019, 9, 6006.
[11] For selected reviews, see: (a) Cao, M.-Y.; Ren, X.; Lu, Z. Tetrahedron Lett. 2015, 56, 3732. (b) Chen, J.-R.; Yu, X.-Y.; Xiao, W.-J. Synthesis 2015, 47, 604. (c) Koike, T.; Akita, M. Acc. Chem. Res. 2016, 49, 1937. (d) Koike, T.; Akita, M. Chem 2018, 4, 409. (e) Li, W.; Xu, W.; Xie, J.; Yu, S.; Zhu, C. Chem. Soc. Rev. 2018, 47, 654 (f) Wu, X.; Wu, S.; Zhu, C. Tetrahedron Lett. 2018, 59, 1328.

[12] (a) Yan, M.; Kawamata, Y.; Baran, P. S. Chem. Rev. 2017, 117, 13230. (b) Zhang, Z.; Ye, J.-H.; Wu, D.-S.; Zhou, Y.-Q.; Yu, D.-G. Chem. Asian J. 2018, 13, 2292. (c) Peshkov, V. A.; Pereshivko, O. P.; Nechaev, A. A; Peshkov, A. A.; Vander Eycken, E. V. Chem. Soc. Rev. 2018, 47, 3861. (d) Tortajada, A.; Juliá-Hernández, F.; Börjesson, M.; Moragas, T.; Martin, R. Angew. Chem., Int. Ed. 2018, 57 15948. (e) Yan, S.-S.; Fu, Q.; Liao, L.-L.; Sun, G.-Q.; Ye, J.-H.; Gong, L.; Bo-Xue, Y.-Z.; Yu, D.-G. Coord. Chem. Rev. 2018, 374 439. (f) Cao, Y.; He, X.; Wang, N.; Li, H.-R.; He, L.-N. Chin. J. Chem. 2018, 36, 644. (g) Hou, J.; Li, J.-S.; Wu, J. Asian J. Org. Chem. 2018, 7, 1439. (h) Tan, F.; Yin, G. Chin. J. Chem. 2018, 36, 545. (i) Yeung, C. S. Angew. Chem., Int. Ed. 2019, 58, 5492.

[13] Luan, Y.-X.; Ye, M. Tetrahedron Lett. 2018, 59, 853.

[14] (a) Tominaga, K.-I.; Sasaki, Y. Catal. Commun. 2000, 1, 1. (b) Tominaga, K.-i.; Sasaki, Y. J. Mol. Catal. A: Chem. 2004, 220, 159. (c) Liu, Q.; Wu, L.; Fleischer, I.; Selent, D.; Franke, R.; Jackstell, R.; Beller, M. Chem. - Eur. J. 2014, 20, 6888. (d) Tani, Y.; Kuga, K. Fujihara, T.; Terao, J.; Tsuji, Y. Chem. Commun. 2015, 51, 13020. (e) Gui, Y.-Y.; Hu, N.; Chen, X.-W.; Liao, L.-L.; Ju, T.; Ye, J.-H.; Zhang, Z.; Li, J.; Yu, D.-G. J. Am. Chem. Soc. 2017, 139, 17011.

[15] Seo, H.; Liu, A.-F.; Jamison, T. F. J. Am. Chem. Soc. 2017, 139, 13969.

[16] (a) Evans, D. A.; Bartroli, J.; Shih, L. T. J. Am. Chem. Soc. 1981, 103, 2127. (b) Pandit, N.; Singla, R. K.; Shrivastava, B. Int. J. Med. Chem. 2012, 2012, 159285. (c) Ed.: Acton, Q. A., Oxazolidinones-Advances in Research and Application, Scholarly Editions, Atlanta, U.S., 2012.

[17] Ye, J.-H.; Song, L.; Zhou, W.-J.; Ju, T.; Yin, Z.-B.; Yan, S.-S.; Zhang, Z.; Li, J.; Yu, D.-G. Angew. Chem. Int. Ed. 2016, 55, 10022.

[18] Zhu, L.; Ye, J.-H.; Duan, M.; Qi, X.; Yu, D.-G.; Bai, R.; Lan, Y. Org. Chem. Front. 2018, 5, 633.

[19] Ye, J.-H.; Zhu, L.; Yan, S.-S.; Miao, M.; Zhang, X.-C.; Zhou, W.-J.; Li, J.; Lan, Y.; Yu, D.-G. ACS Catal. $2017,7,8324$.

[20] Wang, M.-Y.; Cao, Y.; Liu, X.; Wang, N.; He, L.-N.; Li, S.-H. Green Chem. 2017, 19, 1240.

[21] Yin, Z.-B.; Ye, J.-H.; Zhou, W.-J.; Zhang, Y.-H.; Ding, L.; Gui, Y.-Y.; Yan, S.-S.; Li, J.; Yu, D.-G. Org. Lett. 2017, 20, 190.

[22] Zhou, W.-J.; Cao, G.-M.; Sen, G.; Zhu, X.-Y.; Gui, Y.-Y.; Ye, J.-H.; Sun, L.; Liao, L.-L.; Li, J.; Yu, D.-G. Angew. Chem., Int. Ed. 2017, $56,15683$.

[23] (a) Sun, L.; Ye, J.-H.; Zhou, W.-J.; Zeng, X.; Yu, D.-G. Org. Lett. 2018, 20, 3049. (b) For a very recent work, see: Sun, S.; Zhou, C.; Yu, J.-T.; Cheng, J. Org. Lett. 2019, DOI: 10.1021/acs.orglett.9b02700.

[24] Yatham, V. R.; Shen, Y.; Martin, R. Angew. Chem., Int. Ed. 2017, 56, 10915.

[25] Hou, J.; Ee, A.; Cao, H.; Ong, H.-W.; Xu, J.-H.; Wu J. Angew. Chem., Int. Ed. 2017, 57, 17220.

[26] Ye, J.-H.; Miao, M.; Huang, H.; Yan, S.-S.; Yin, Z.-B.; Zhou, W.-J.; Yu, D.-G. Angew. Chem., Int. Ed. 2017, 56, 15416.

[27] Senboku, H.; Komatsu, H.; Fujimura, Y.; Tokuda, M. Synlett 2001, $2001,418$.

[28] Yuan, G.-Q.; Jiang, H.-F.; Lin, C.; Liao, S.-J. Electrochim. Acta 2008, 53, 2170.

[29] Li, C.-H.; Yuan, G.-Q.; Ji, X.-C.; Wang, X.-J.; Ye, J.-S.; Jiang, H.-F. Electrochim. Acta 2011, 56, 1529.

[30] For a very recent work on phosphonocarboxylation of alkenes with $\mathrm{CO}_{2}$, see: Fu, Q.; Bo, Z.-Y.; Ye, J.-H.; Ju, T.; Huang, H.; Liao, L.-L.; Yu, D.-G. Nat. Commun. 2019, 10, 3592. 